\title{
The Strength of the Low-Ionization Emission Lines in Type I PNe
}

\author{
A. Riera $^{1,2}$ \\ ${ }^{1}$ Departament de Física i Enginyeria Nuclear; \\ ${ }^{2}$ Departament d'Astronomia i Meteorologia, Barcelona
}

I have performed a statistical analysis on a group of emission line ratios of a sample of PNe. The optical data was taken from ELCAT2 Catalogue

(htpp://www.stsci.edu/pub/elcat). Some observations of $\mathrm{H}-\mathrm{H}$ objects were also included for comparison.

The main objective is to give insight into the properties that characterize the spectra of PNe. With this aim, I have plotted a group of diagrams based on line ratios involving [OI] $\lambda 6300 \AA,[\mathrm{OII}] \lambda 3727 \AA,[\mathrm{OIII}] \lambda 4363,5007 \AA,[\mathrm{NI}] \lambda 5200 \AA,[\mathrm{NII}] \lambda 5755,6583$ $\AA$, [SII] $\lambda 6717,6731 \AA$ and HeII $\lambda 4686 \AA$ as well as HI Balmer emission lines. Both PNe groups show different low-ionization spectra. The [SII] $(6717+6731) / \mathrm{H} \alpha$ vs. [OI] $6300 / \mathrm{H} \alpha$ diagram shows the cleanest separation, with three distinct zones corresponding to each group. The non-Type I PNe have the lowest values, while $\mathrm{HH}$ objects are located in the upper-right corner of the diagram. The Type I PNe adopt intermediate values.

The diagrams illustrate that: (i) it is possible to distinguish the spectra of a non-Type I and Type I PNe by some $\mathrm{N}$ independent ratios; and (ii) the spectra of the Type I PNe and the $\mathrm{HH}$ objects share some properties concerning the low-ionization emission lines.

There are some explanations that can be regarded as responsible for the spectral differences between the non-Type I and the Type I PNe: (i) the presence of shocked regions inside the latter group, (ii) the presence of filaments and/or dense condensations in the Type I PNe where the charge exchange reactions are enhanced (see Riera et al. also presented here). 